\title{
Deleterious EPCAM Gene Mutation
}

National Cancer Institute

\section{Source}

National Cancer Institute. Deleterious EPCAM Gene Mutation. NCI Thesaurus. Code C136970.

A change in the nucleotide sequence of the EPCAM gene that is associated with increased risk of disease. 